EPJ manuscript No.

(will be inserted by the editor)

\title{
ALICE perspectives for the study of charm and beauty energy loss at the LHC
}

\author{
A. Dainese for the ALICE Collaboration \\ INFN - LNL, Legnaro (Padova), Italy
}

Received: August 20, 2019

\begin{abstract}
At LHC energy, heavy quarks will be abundantly produced and the design of the ALICE detector will allow us to study their production using several channels. The expected heavy-quark in-medium energy loss in nucleus-nucleus collisions at the LHC is calculated within a model, that is compared to the available heavy-quark quenching measurements at RHIC. The nuclear modification factors and heavy-to-light ratios of charm and beauty mesons are considered. The capability of the ALICE experiment for addressing this phenomenology is discussed.
\end{abstract}

PACS. 2 5.75.-q, 14.65.Dw, 13.25.Ft

\section{Introduction}

The ALICE experiment [1,2] will study $\mathrm{Pb}-\mathrm{Pb}$ collisions at the LHC, with a centre-of-mass energy $\sqrt{s_{\mathrm{NN}}}=5.5 \mathrm{TeV}$, in order to investigate the properties of QCD matter at energy densities of up to several hundred times the density of atomic nuclei. Under these conditions a deconfined state of quarks and gluons is expected to be formed.

The measurement of open charm and open beauty production allows to investigate the mechanisms of heavyquark production and propagation in the hot and dense medium formed in the collision. In particular, mediuminduced partonic energy loss of heavy quarks has recently become one of the most exciting and disputed issues within high-energy heavy-ion physics, after the observation at RHIC of a large suppression in the production of hightransverse-momentum electrons from heavy-flavour decays. In Section 2 we describe these experimental results and we compare them to a model implementation of a particular energy loss calculation. Within this model we then obtain predictions for relevant charm and beauty energy loss observables at LHC energy. After providing, in Section [3 a general overview of the heavy-flavour capabilities of the ALICE detector, we present the expected sensitivity in the measurement of energy loss effects, in Section 4.

\section{Heavy-quark energy loss from RHIC to LHC}

Believed to be the main origin of the jet quenching effects observed 3 in nucleus-nucleus collisions at RHIC energy $\sqrt{s_{\mathrm{NN}}}=62-200 \mathrm{GeV}$, parton energy loss via gluonradiation is expected to depend on the properties (gluon density and volume) of the medium and on the properties (colour charge and mass) of the 'probe' parton. Gluons would lose more energy than quarks due to the stronger colour coupling. In addition, charm and beauty quarks are qualitatively different probes with respect to light partons, since their energy loss is expected to be reduced, as a consequence of a mass-dependent restriction in the phase-space into which gluon radiation can occur [5,6,7. 8 .

Quenching effects for heavy quarks can be estimated by supplementing perturbative QCD calculations of the baseline $p_{\mathrm{t}}$ distributions with in-medium energy loss. Here, we consider the particular radiative energy loss calculation that is implemented in the BDMPS formalism 9. The energy loss probability distributions (quenching weights) were computed for light quarks and gluons in [10] and for heavy quarks in 11. They depend on the medium transport coefficient $\hat{q}$, the average transverse momentum squared transferred from probe parton to the scattering centres in the medium per unit mean free path, and on the in-medium path length $L$ of the probe parton. The collision geometry is included by evaluating $\hat{q}$ and $L$ on a parton-by-parton level, using a Glauber-model based description of the local $\hat{q}$ profile in the transverse plane 12 . The parton-averaged $\langle\hat{q}\rangle$ value (hereafter called $\hat{q}$ for brevity) is chosen in order to reproduce the factor 4-5 suppression measured for the nuclear modification factor

$$
R_{\mathrm{AA}}\left(p_{\mathrm{t}}\right)=\frac{1}{\left\langle N_{\text {coll }}\right\rangle} \times \frac{\mathrm{d}^{2} N_{\mathrm{AA}} / \mathrm{d} p_{\mathrm{t}} \mathrm{d} \eta}{\mathrm{d}^{2} N_{\mathrm{pp}} / \mathrm{d} p_{\mathrm{t}} \mathrm{d} \eta}
$$

of light-flavour particles in central $\mathrm{Au}-\mathrm{Au}$ collisions at $\sqrt{s_{\mathrm{NN}}}=200 \mathrm{GeV}$. The range favoured by the data is $\hat{q}=4-14 \mathrm{GeV}^{2} / \mathrm{fm}$ [13,14.

Heavy-quark energy loss is presently studied at RHIC using measurements of the nuclear modification factor $R_{\mathrm{AA}}$ of 'non-photonic' ( $\gamma$-conversion- and $\pi^{0}$-Dalitz-subtracted $)$ 

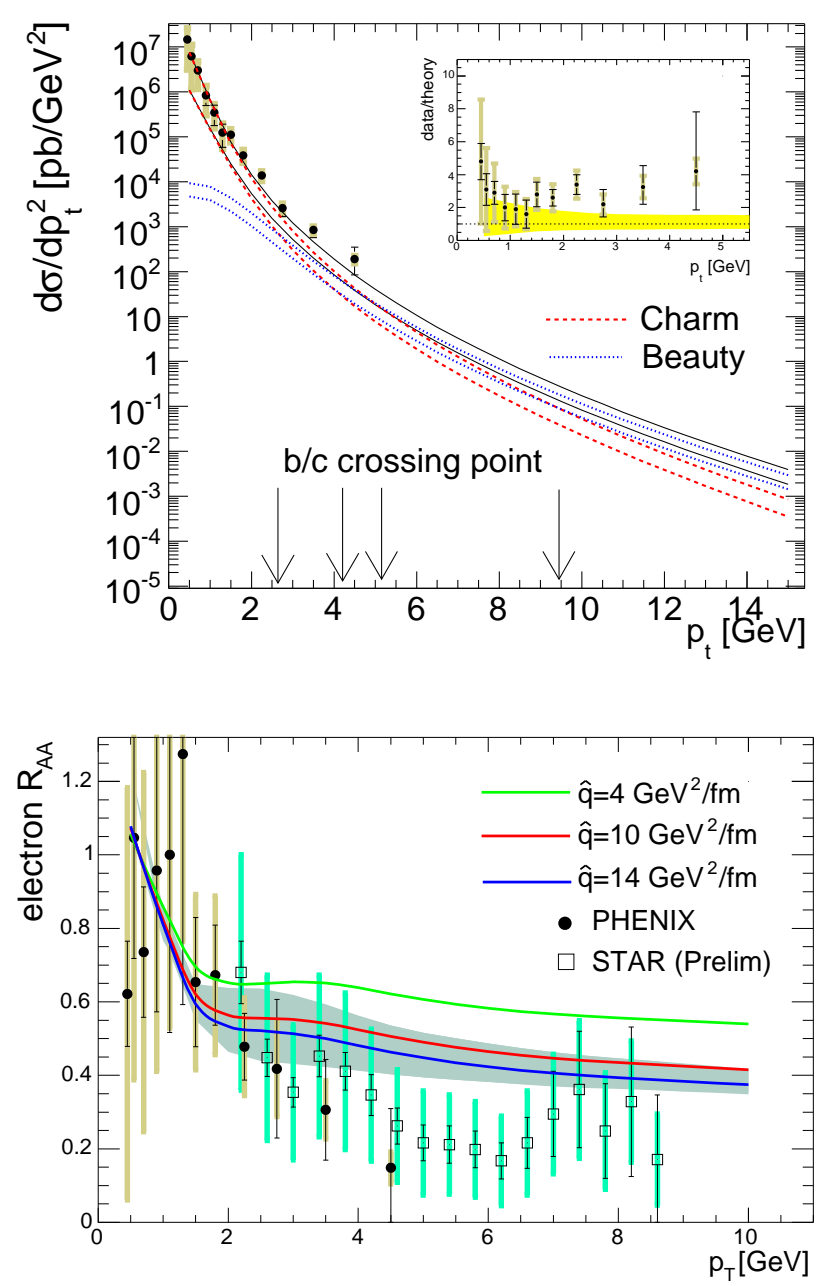

Fig. 1. From 15]. Upper panel: comparison of the FONLL calculation of single inclusive electrons [16] to data from [17] pp collisions at $\sqrt{s}=200 \mathrm{GeV}$. Upper and lower lines are estimates of theoretical uncertainties, obtained by varying scales and masses. Lower panel: $R_{\mathrm{AA}}$ of electrons in central $\mathrm{Au}-\mathrm{Au}$ collisions 18 19. Curves indicate the suppression for different opacities of the produced matter. The shaded band indicates the theoretical uncertainty of the perturbative baseline for $\hat{q}=14 \mathrm{GeV}^{2} / \mathrm{fm}$.

single electrons. Since this is an inclusive measurement, with charm decays dominating at low $p_{\mathrm{t}}$ and beauty decays dominating at high $p_{\mathrm{t}}$, the comparison with massdependent energy loss predictions should rely on a solid and data-validated pp baseline. Such baseline is still lacking at the moment. The state-of-the-art perturbative predictions (FONLL), usually employed as a baseline, indicate that, in pp collisions, charm decays dominate the electron $p_{\mathrm{t}}$ spectrum up to about $5 \mathrm{GeV}$ 15. However, there is a large perturbative uncertainty on the position in $p_{\mathrm{t}}$ of the c-decay/b-decay crossing point: depending on the choice of the factorization and renormalization scales this position can vary from 3 to $9 \mathrm{GeV}$ [15], as illustrated in Fig. 11 (upper panel). In addition, as shown in the insert, the calculation underpredicts the non-photonic electron spectrum measured in pp collisions [17.

The most recent data by PHENIX [18] and STAR [19] on the nuclear modification factor $R_{\mathrm{AA}}^{\mathrm{e}}$ of non-photonic electrons in central $\mathrm{Au}-\mathrm{Au}$ collisions at $\sqrt{s_{\mathrm{NN}}}=200 \mathrm{GeV}$ are shown in Fig. 1 (lower panel). The theoretical expectation is superimposed to the data, with the uncertainty on the medium density (curves for $\hat{q}=4,10,14 \mathrm{GeV}^{2} / \mathrm{fm}$ ) and the perturbative uncertainty, obtained by varying the values of the scales and of the $c$ and $b$ quark masses (shaded band associated to the $\hat{q}=14 \mathrm{GeV}^{2} /$ fm curve) [15]. The calculation tends to overpredict the measured $R_{\mathrm{AA}}$. It has recently been argued 20] that parton energy loss would have a significant collisional contribution, comparable to the radiative one. Although the quantitative relevance of the collisional contribution is still debated [21], the effect has been included in heavy-quark energy loss calculations 20. Yet, the large suppression measured for $R_{\mathrm{AA}}^{\mathrm{e}}$ can not be well reproduced [20].

It is important to note that, in general, the perturbative uncertainty in calculating the partonic baseline spectrum is comparable to the model-intrinsic uncertainty in determining $\hat{q}$. Thus, the strongest limitation to the sensitivity in the theory-data comparison comes from the inability of the RHIC experiments, in their present detector setup, to disentangle the charm and beauty contributions to single electrons.

Heavy quarks will be produced with large cross sections at LHC energy and the experiments will be equipped with detectors optimized for the separation of charm and beauty decay vertices. Thus, it will possible to carry out a direct comparison of the attenuation of light-flavour hadrons, D mesons, and B mesons.

The expected nuclear modification factors $R_{\mathrm{AA}}$ were calculated in 11] exploring a large range in the medium density for central $\mathrm{Pb}-\mathrm{Pb}$ collisions at $\sqrt{s_{\mathrm{NN}}}=5.5 \mathrm{TeV}$ : $25<\hat{q}<100 \mathrm{GeV}^{2} /$ fm. Figure 2 (thick lines) shows the results for the heavy-to-light ratios of D and B mesons [11, defined as the ratios of the nuclear modification factors of $\mathrm{D}(\mathrm{B})$ mesons to that of light-flavour hadrons $(h): R_{\mathrm{D}(\mathrm{B}) / h}=$ $R_{\mathrm{AA}}^{\mathrm{D}(\mathrm{B})} / R_{\mathrm{AA}}^{h}$. The effect of the mass is illustrated by artificially neglecting the mass dependence of parton energy loss (thin curves). The enhancement above unity that persists in the $m_{\mathrm{c}(\mathrm{b})}=0$ cases is mainly due to the colourcharge dependence of energy loss, since at LHC energy most of the light-flavour hadrons will originate from a gluon parent. The calculation results indicate that, for $\mathrm{D}$ mesons, the mass effect is small and limited to the region $p_{\mathrm{t}} \lesssim 10 \mathrm{GeV}$, while for B mesons a large enhancement can be expected up to $20 \mathrm{GeV}$. Therefore, the comparison of the high- $p_{\mathrm{t}}$ suppression for D mesons and for light-flavour hadrons would test the colour-charge dependence (quark parent vs. gluon parent) of parton energy loss, while the comparison for B mesons and for light-flavour hadrons would test its mass dependence [1]. 

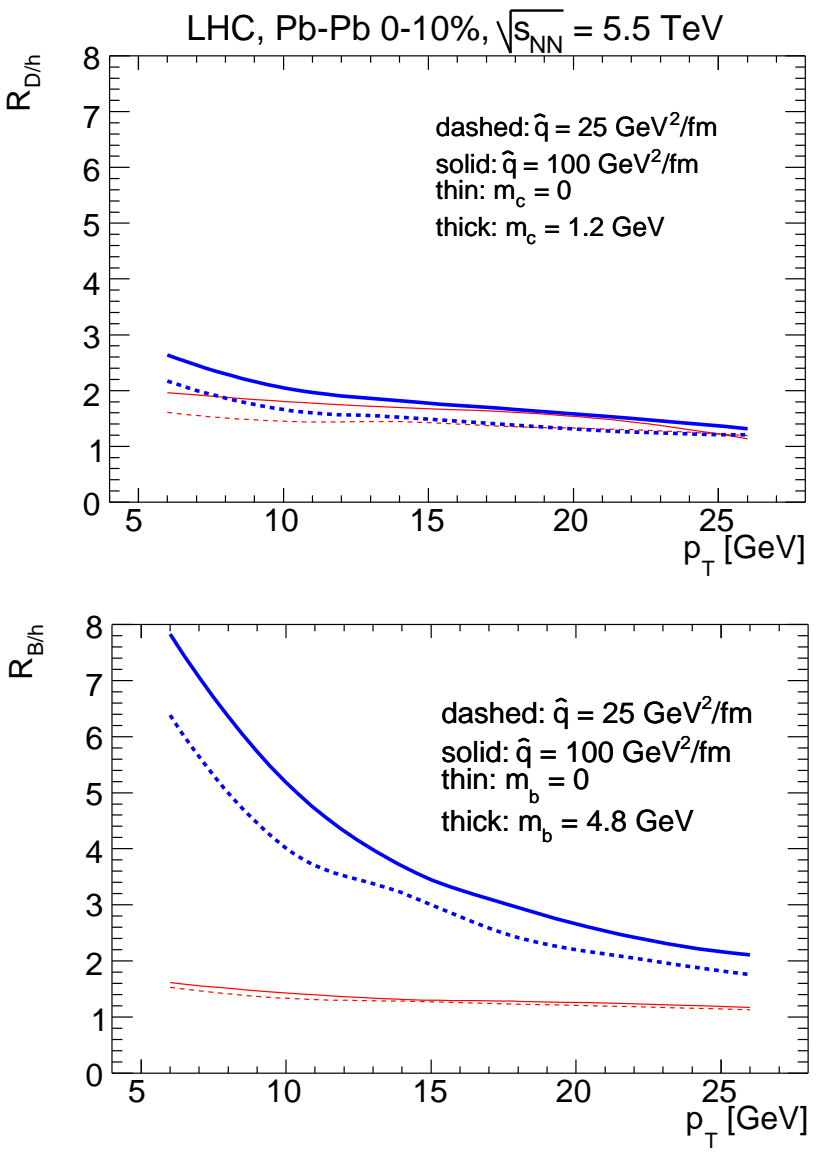

Fig. 2. Heavy-to-light ratios for D (upper panel) and B (lower panel) mesons for the case of realistic heavy-quark masses and for a case study in which the quark mass dependence of parton energy loss is neglected [1].

\section{Heavy-flavour detection in ALICE}

The ALICE experimental setup [1] was designed so as to allow the detection of $\mathrm{D}$ and $\mathrm{B}$ mesons in the highmultiplicity environment of central $\mathrm{Pb}-\mathrm{Pb}$ collisions at LHC energy, where up to several thousand charged particles might be produced per unit of rapidity. The heavyflavour capability of the ALICE detector is provided by:

- Tracking system; the Inner Tracking System (ITS), the Time Projection Chamber (TPC) and the Transition Radiation Detector (TRD), embedded in a magnetic field of $0.5 \mathrm{~T}$, allow track reconstruction in the pseudorapidity range $-0.9<\eta<0.9$ with a momentum resolution better than $2 \%$ for $p_{\mathrm{t}}<20 \mathrm{GeV}$ and a transverse impact parameter ${ }^{1}$ resolution better than $60 \mu \mathrm{m}$ for $p_{\mathrm{t}}>1 \mathrm{GeV}$ (the two innermost layers of the ITS are equipped with silicon pixel detectors $)^{2}$.

1 The transverse impact parameter, $d_{0}$, is defined as the distance of closest approach of the track to the interaction vertex, in the plane transverse to the beam direction.

2 Note that, for pp collisions, the impact parameter resolution may be slightly worse, due to the larger transverse size of
- Particle identification system; charged hadrons are identified via $\mathrm{d} E / \mathrm{d} x$ in the TPC and in the ITS and via time-of-flight measurement in the Time Of Flight (TOF) detector; electrons are separated from charged pions in the dedicated TRD, and in the TPC; muons are identified in the forward muon spectrometer covering in acceptance the range $-4<\eta<-2.5$.

Detailed studies 2, based on full simulation of the detector and of the background sources, have shown that ALICE has a good potential to carry out a rich heavyflavour physics programme. Several analyses aimed at investigating quenching effects for $\mathrm{c}$ and $\mathrm{b}$ quarks are being prepared. Here, we focus on the two most advanced studies in the central barrel: exclusive reconstruction of charm particles, in the $\mathrm{D}^{0} \rightarrow \mathrm{K}^{-} \pi^{+}$decay channel, and inclusive measurement of beauty particles, in the semi-electronic decay channels $\mathrm{B} \rightarrow \mathrm{e}+X$. Excellent performance is also expected for the measurement of beauty production at forward rapidity in the semi-muonic decay channels [2] 22. In this context, the study of the single-inclusive muon distribution in the range $20 \lesssim p_{\mathrm{t}} \lesssim 50 \mathrm{GeV}$ is a new promising tool to address energy loss effects for b quarks. At LHC energy, single muons are dominated by decays of $b$ quarks, expected to strongly interact with the medium, for $3 \lesssim p_{\mathrm{t}} \lesssim 30 \mathrm{GeV}$ and by decays of weakly-interacting, thus 'medium-blind', $\mathrm{W}^{ \pm}$bosons for $p_{\mathrm{t}} \gtrsim 30 \mathrm{GeV} 23$. Therefore, the position in $p_{\mathrm{t}}$ of the crossing point between bdecay and $\mathrm{W}$-decay muons should be sensitive to the inmedium energy loss of b quarks.

For all studies a multiplicity of $\mathrm{d} N_{\mathrm{ch}} / \mathrm{d} y=6000$ was assumed for central $\mathrm{Pb}-\mathrm{Pb}$ collisions ${ }^{3}$. We report the results corresponding to the expected statistics collected by ALICE per LHC year: $10^{7}$ central $\left(0-5 \% \sigma^{\text {inel }}\right) \mathrm{Pb}-\mathrm{Pb}$ events at $\mathcal{L}_{\mathrm{Pb}-\mathrm{Pb}}=10^{27} \mathrm{~cm}^{-2} \mathrm{~s}^{-1}$ and $10^{9}$ pp events at $\mathcal{L}_{\mathrm{pp}}^{\mathrm{ALICE}}=$ $5 \times 10^{30} \mathrm{~cm}^{-2} \mathrm{~s}^{-1}$, in the barrel detectors.

\subsection{Charm production: $\mathrm{D}^{0} \rightarrow \mathrm{K}^{-} \pi^{+}$}

One of the most promising channels for open charm detection is the $\mathrm{D}^{0} \rightarrow \mathrm{K}^{-} \pi^{+}$decay which has a branching ratio (BR) of about $3.8 \%$. Based on next-to-leading order pQCD calculations [4, the expected production yields $(\mathrm{BR} \times \mathrm{d} N / \mathrm{d} y$ at $y=0)$ for $\mathrm{D}^{0}\left(+\overline{\mathrm{D}^{0}}\right)$ mesons decaying in a $\mathrm{K}^{\mp} \pi^{ \pm}$pair in central $\mathrm{Pb}-\mathrm{Pb}\left(0-5 \% \sigma^{\text {inel }}\right)$ at $\sqrt{s_{\mathrm{NN}}}=5.5 \mathrm{TeV}$ and in pp collisions at $\sqrt{s}=14 \mathrm{TeV}$ are $5.3 \times 10^{-1}$ and $7.5 \times 10^{-4}$ per event, respectively [2].

Figure 3 shows a sketch of the decay: the main feature of this topology is the presence of two tracks with impact parameters $d_{0} \sim 100 \mu \mathrm{m}$. The detection strategy [24] to cope with the large combinatorial background from the underlying event is based on:

1. selection of displaced-vertex topologies, i.e. two tracks with large impact parameters and small pointing angle

the beam at the ALICE interaction point. This is taken into account in the studies presented here.

3 This value of the multiplicity can be taken as a conservative assumption, since extrapolations based on RHIC data predict $\mathrm{d} N_{\mathrm{ch}} / \mathrm{d} y=2000-3000$. 


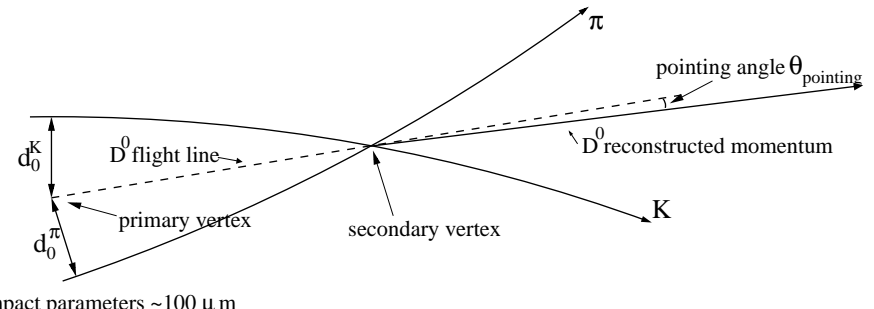

impact parameters $\sim 100 \mu \mathrm{m}$

Fig. 3. Schematic representation of the $\mathrm{D}^{0} \rightarrow \mathrm{K}^{-} \pi^{+}$decay with the impact parameters $\left(d_{0}\right)$ and the pointing angle $\left(\theta_{\text {pointing }}\right)$.

$\theta_{\text {pointing }}$ between the $\mathrm{D}^{0}$ momentum and flight-line (see sketch in Fig. 31);

2. identification of the $\mathrm{K}$ track in the TOF detector;

3. invariant-mass analysis.

This strategy was optimized separately for pp and $\mathrm{Pb}-$ $\mathrm{Pb}$ collisions, as a function of the $\mathrm{D}^{0}$ transverse momentum 25. 2. As shown in Fig. 4 the accessible $p_{\mathrm{t}}$ range is 1-20 GeV for $\mathrm{Pb}-\mathrm{Pb}$ and $0.5-20 \mathrm{GeV}$ for $\mathrm{pp}$, with a statistical error better than 15-20\%. The systematic errors (acceptance and efficiency corrections, centrality selection for $\mathrm{Pb}-\mathrm{Pb}$ ) are expected to be smaller than $20 \%$. More details are given in Refs. 25 2.

\subsection{Beauty production: $\mathrm{B} \rightarrow \mathrm{e}+X$}

The production of open beauty can be studied by detecting the semi-electronic decays of beauty hadrons, mostly $\mathrm{B}$ mesons. Such decays have a branching ratio of $\simeq 10 \%$ (plus $10 \%$ from cascade decays $\mathrm{b} \rightarrow \mathrm{c} \rightarrow \mathrm{e}$, that only populate the low- $p_{\mathrm{t}}$ region in the electron spectrum). The expected yields $(\mathrm{BR} \times \mathrm{d} N / \mathrm{d} y$ at $y=0)$ for $\mathrm{b} \rightarrow \mathrm{e}+X$ plus $\mathrm{b} \rightarrow \mathrm{c}(\rightarrow \mathrm{e}+X)+X^{\prime}$ in central $\mathrm{Pb}-\mathrm{Pb}\left(0-5 \% \sigma^{\text {inel }}\right)$ at $\sqrt{s_{\mathrm{NN}}}=5.5 \mathrm{TeV}$ and in in pp collisions at $\sqrt{s}=14 \mathrm{TeV}$ are $1.8 \times 10^{-1}$ and $2.8 \times 10^{-4}$ per event, respectively 2 .

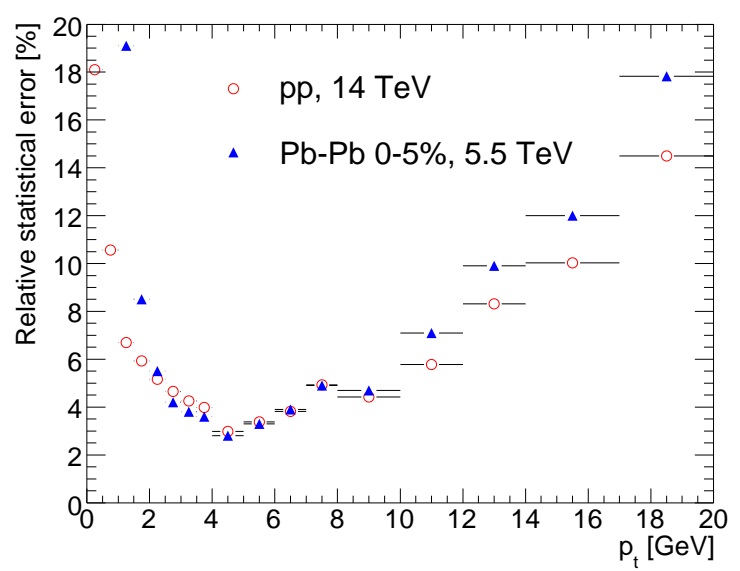

Fig. 4. Expected relative statistical errors on the measurement of the $\mathrm{D}^{0}$ production cross section, in $0-5 \%$ central $\mathrm{Pb}-\mathrm{Pb}$ collisions and in pp collisions.
The main sources of background electrons are: (a) decays of D mesons; (b) neutral pion Dalitz decays $\pi^{0} \rightarrow$ $\gamma \mathrm{e}^{+} \mathrm{e}^{-}$and decays of light mesons (e.g. $\rho$ and $\left.\omega\right) ;(\mathrm{c})$ conversions of photons in the beam pipe or in the inner detector layer and (d) pions misidentified as electrons. Given that electrons from beauty have average impact parameter $d_{0} \simeq 500 \mu \mathrm{m}$ and a hard momentum spectrum, it is possible to obtain a high-purity sample with a strategy that relies on:

1. electron identification with a combined $\mathrm{d} E / \mathrm{d} x$ (TPC) and transition radiation selection, which is expected to reduce the pion contamination by a factor $10^{4}$;

2. impact parameter cut to reject misidentified $\pi^{ \pm}$and $\mathrm{e}^{ \pm}$from Dalitz decays and $\gamma$ conversions (the latter have small impact parameter for $\left.p_{\mathrm{t}} \gtrsim 1 \mathrm{GeV}\right)$;

3. $p_{\mathrm{t}}$ cut to reject electrons from charm decays.

As an example, with $d_{0}>200 \mu \mathrm{m}$ and $p_{\mathrm{t}}>2 \mathrm{GeV}$, the expected statistics of electrons from b decays is $8 \times 10^{4}$ for $10^{7}$ central $\mathrm{Pb}-\mathrm{Pb}$ events, allowing the measurement of electron-level $p_{\mathrm{t}}$-differential cross section in the range $2<$ $p_{\mathrm{t}}<20 \mathrm{GeV}$. The residual contamination of about $10 \%$ of electrons from prompt charm decays, from misidentified charged pions and $\gamma$-conversion electrons can be evaluated and subtracted using a Monte Carlo simulation tuned to reproduce the measured cross sections for pions and $\mathrm{D}^{0}$ mesons. In Fig. [5 we show the expected relative statistical errors on the measurement of the cross section of electrons from beauty decays. A Monte-Carlo-based procedure can then be used to compute, from the electron-level cross section, the B-level cross section $\mathrm{d} \sigma^{\mathrm{B}}\left(p_{\mathrm{t}}>p_{\mathrm{t}}^{\min }\right) / \mathrm{d} y[2$. The covered range is $2<p_{\mathrm{t}}^{\mathrm{min}}<30 \mathrm{GeV}$.

\section{Sensitivity to energy loss}

We investigated the possibility of using the described charm and beauty measurements to study the dependences of parton energy loss. This study could be carried out by measuring:

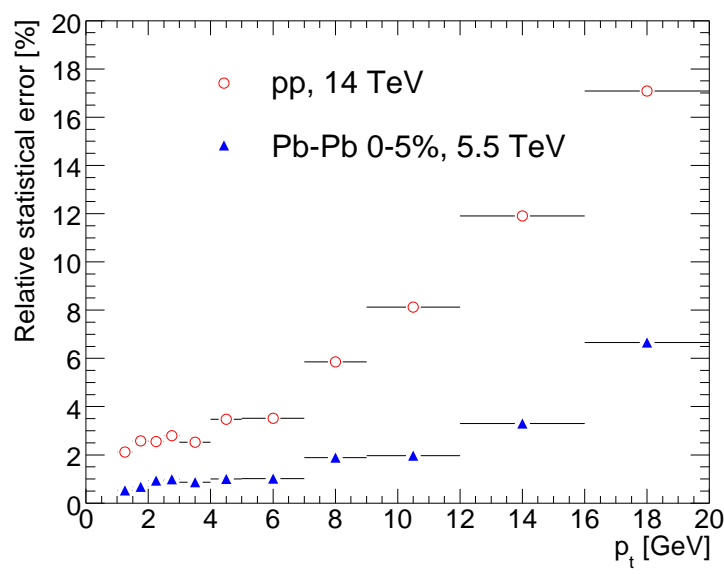

Fig. 5. Expected relative statistical errors on the measurement of the production cross section of B-decay electrons, in $0-5 \%$ central $\mathrm{Pb}-\mathrm{Pb}$ collisions and in pp collisions. 
- the nuclear modification factor of D mesons as a function of transverse momentum, $R_{\mathrm{AA}}^{\mathrm{D}}\left(p_{\mathrm{t}}\right)$;

- the nuclear modification factor of b-decay electrons as a function of transverse momentum, $R_{\mathrm{AA}}^{\text {efrom B }}\left(p_{\mathrm{t}}\right)$;

- the heavy-to-light ratio, $R_{\mathrm{D} / h}\left(p_{\mathrm{t}}\right)$, defined as the ratio of the nuclear modification factors of $\mathrm{D}$ mesons and of charged light-flavoured hadrons.

We compare the expected experimental errors on these observables to recent theoretical predictions parton energy loss 11.

The expected performance for the measurement of the nuclear modification factor for $\mathrm{D}^{0}$ mesons is reported in Fig. [6] Only nuclear shadowing and parton intrinsic transverse-momentum broadening are included (no energy loss). The reported statistical (bars) and systematic (shaded areas) errors are obtained combining the errors for $\mathrm{Pb}-\mathrm{Pb}$ and pp collisions and assuming that the contributions due to cross section normalization, feed-down from beauty decays and, partially, acceptance/efficiency corrections will cancel out in the ratio. An expected uncertainty of about $12 \%$ [2] introduced in the extrapolation of the pp results from $14 \mathrm{TeV}$ to $5.5 \mathrm{TeV}$ by pQCD is also included.

The effect of shadowing, included via the EKS98 parametrisation [26], is visible as a suppression of $R_{\mathrm{AA}}$ at low transverse momenta, corresponding to small Bjorken $x$. The effect is negligible for $p_{\mathrm{t}}>6-7 \mathrm{GeV}$. Since there is significant uncertainty on the magnitude of shadowing in the low- $x$ region, we studied the effect of such uncertainty on $R_{\mathrm{AA}}$ by varying the nuclear modification of parton distribution functions. Also in the case of shadowing $50 \%$ stronger than in EKS98 (curve labelled ' $\mathrm{c}$ '), we find $R_{\mathrm{AA}}>0.95$ for $p_{\mathrm{t}}>8 \mathrm{GeV}$. Under these assumptions, for $p_{\mathrm{t}}>8 \mathrm{GeV}$ only parton energy loss is expected to affect the nuclear modification factor of D mesons.

Figure $\mathbf{Z}$ presents the predicted 11] nuclear modification factor without $(\hat{q}=0)$ and with energy loss (the bands correspond to the range $25<\hat{q}<100 \mathrm{GeV}^{2} / \mathrm{fm}$ ). The effect of the charm mass on energy loss is included for the thick-line band $\left(m_{\mathrm{c}}=1.2 \mathrm{GeV}\right)$ and not included for the thin-line band $\left(m_{\mathrm{c}}=0\right)$. The small difference between the two bands indicates that, with respect to energy loss, charm behaves essentially as a massless quark. The

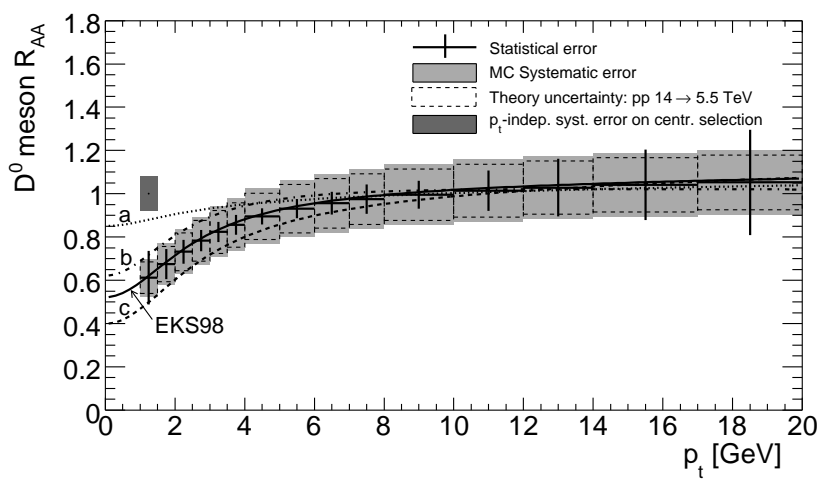

Fig. 6. Illustration of the experimental uncertainties on the $R_{\mathrm{AA}}$ of $\mathrm{D}^{0}$ mesons (no energy loss).

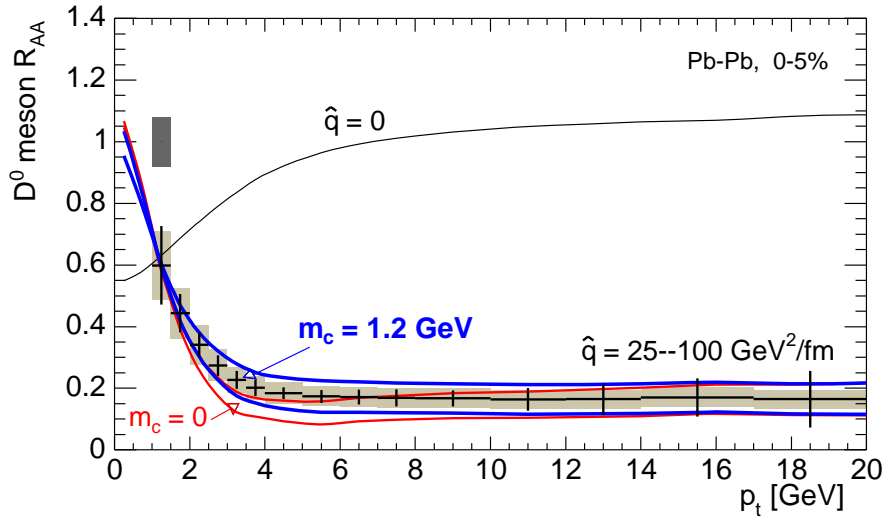

Fig. 7. Nuclear modification factor for $\mathrm{D}^{0}$ mesons with shadowing. The two bands represent theoretical predictions with and without the effect of the charm mass in the energy loss for the medium transport coefficient range $\hat{q}=25-$ $100 \mathrm{GeV}^{2} / \mathrm{fm}$ 11. Errors corresponding to the centre of the prediction band for $m_{\mathrm{c}}=1.2 \mathrm{GeV}$ are shown: bars = statistical, shaded area $=$ systematic. The normalization error is shown by the box at $R_{\mathrm{AA}}=1$.

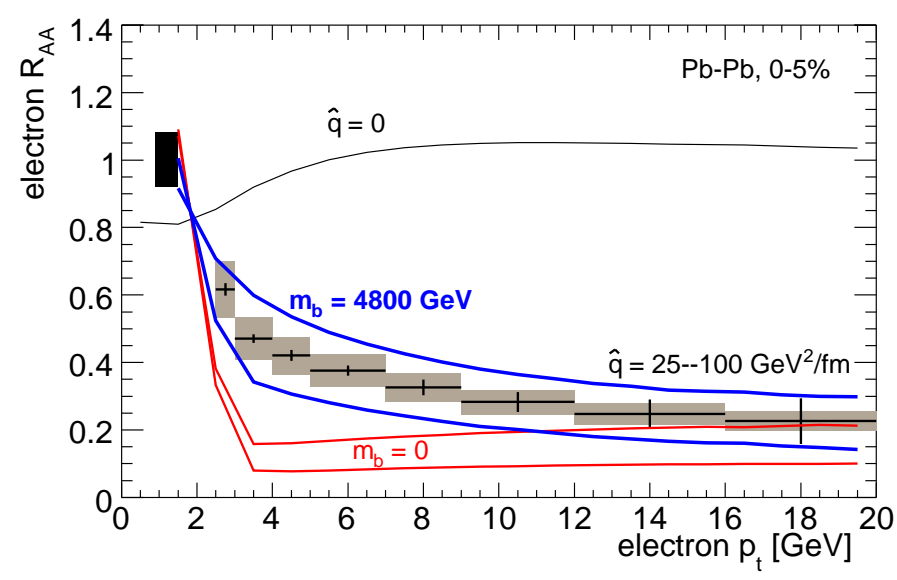

Fig. 8. Nuclear modification factor for b-decay electrons with shadowing. The two bands represent theoretical predictions with and without the effect of the beauty mass in the energy loss for the medium transport coefficient range $\hat{q}=25-$ $100 \mathrm{GeV}^{2} / \mathrm{fm}$ [1]. Errors are defined as for Fig. 7

estimated uncertainties for the measurement of $R_{\mathrm{AA}}^{\mathrm{D}}$ are reported here for the case with energy loss. The bars represent the statistical errors, while the shaded areas represent the quadratic sum of the systematic error from Monte Carlo corrections $(\approx 15 \%)$ and that from the $\sqrt{s}$ extrapolation of the pp measurement from $14 \mathrm{TeV}$ to $5.5 \mathrm{TeV}$ $(\approx 12 \%)$. Owing to the predicted suppression of about a factor 5 for $p_{\mathrm{t}} \gtrsim 5 \mathrm{GeV}$, the relative statistical errors in $\mathrm{Pb}-\mathrm{Pb}$ are larger by more than a factor 2 , with respect to the case of no suppression, and they become the dominant contribution to the statistical error on $R_{\mathrm{AA}}^{\mathrm{D}}$.

The expected performance for the measurement of the nuclear modification factor of electrons from B-meson decays is shown in Fig. [ along with the predicted suppres- 


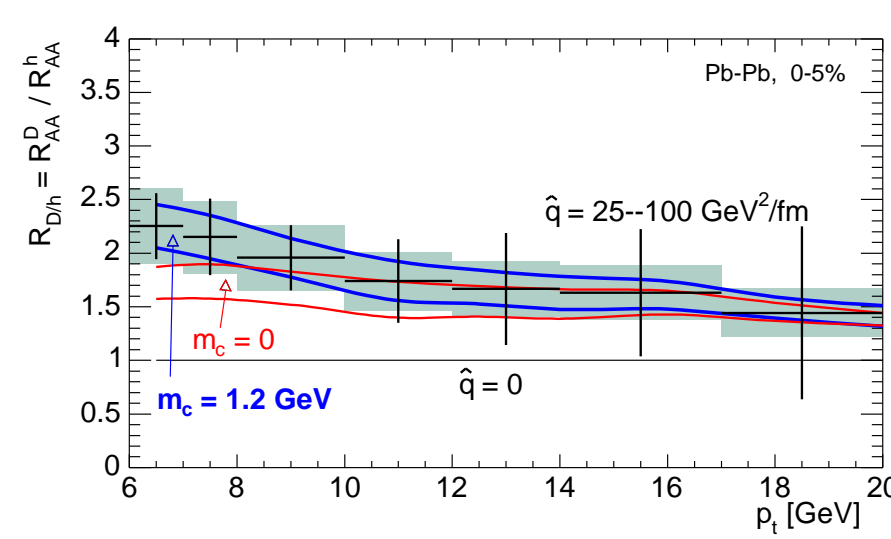

Fig. 9. Ratio of the nuclear modification factors for $\mathrm{D}^{0}$ mesons and for charged hadrons. Errors corresponding to the centre of the prediction band for $m_{\mathrm{c}}=1.2 \mathrm{GeV}$ are shown: bars $=$ statistical, shaded area $=$ systematic.

sion with and without the effect of the beauty mass in the energy loss. Contrary to the case of charm, the suppression is strongly reduced in the range $2 \lesssim p_{\mathrm{t}} \lesssim 15 \mathrm{GeV}$ due the large value of the $b$ mass. As the charm case, the bars represent the statistical errors, while the shaded areas represent the quadratic sum of the systematic error from Monte Carlo corrections $(\approx 15 \%)$ and that from the $\sqrt{s}$ extrapolation of the pp measurement from $14 \mathrm{TeV}$ to $5.5 \mathrm{TeV}(\approx 8 \%)$.

As discussed in Section 2 the comparison of the high$p_{\mathrm{t}}$ suppression of charm-quark-originated mesons and gluonoriginated hadrons may be the tool best suited to single out the predicted colour-charge dependence of $\mathrm{QCD}$ energy loss. The ALICE sensitivity to the heavy-to-light ratio $R_{\mathrm{D} / h}$ in the range $5<p_{\mathrm{t}}<20 \mathrm{GeV}$ is presented in Fig. 9 Like for the case of $R_{\mathrm{AA}}^{\mathrm{D}}$, the two bands correspond to including or not including the effect of the c-quark mass for a medium transport coefficient in the range $25-100 \mathrm{GeV}^{2} / \mathrm{fm}$. For $10<p_{\mathrm{t}}<20 \mathrm{GeV}$, the two bands coincide and predict $R_{\mathrm{D} / h} \approx 1.5$, i.e., about $50 \%$ smaller suppression for $\mathrm{D}$ mesons relative to lightflavoured hadrons. Many of the systematic uncertainties on $R_{\mathrm{D} / h}$ cancel out (centrality selection and, partially, acceptance/efficiency corrections and energy extrapolation by pQCD) since $R_{\mathrm{D} / h}$ is essentially a double ratio. The residual systematic error is estimated to be of about $15 \%$. We assumed the statistical error on $R_{\mathrm{AA}}^{h}$ to be negligible with respect to that on $R_{\mathrm{AA}}^{\mathrm{D}}$ for $p_{\mathrm{t}}<20 \mathrm{GeV}$. The resulting statistical errors on $R_{\mathrm{D} / h}$ are quite large for $p_{\mathrm{t}} \gtrsim 15 \mathrm{GeV}$. However, at lower momenta, the measurement of the compatibility (or incompatibility) of $R_{\mathrm{D} / h}$ with unity appears to be feasible. A procedure to use the electrons from beauty decays to estimate the heavy-tolight ratio of B mesons (lower panel of Fig. 2) is currently under study.

\section{Conclusions}

Heavy-quark quenching studies have become one of the most intriguing topics in heavy-ion physics, with the observation at RHIC of a large suppression for heavy-flavour decay electrons, which is at present not clearly understood within parton energy loss models.

At LHC energy, charm and beauty production cross sections are expected to be larger by factors of approximately 10 and 100, respectively, with respect to RHIC energy. The ALICE experiment will be equipped with highresolution silicon vertex detectors, allowing direct and precise measurements of the main observables that are suggested to be sensitive to the colour-charge and mass dependences of parton energy loss.

\section{References}

1. ALICE Collaboration, Physics Performance Report Vol. I, J. Phys. G 30, 1517 (2003) (CERN/LHCC 2003049).

2. ALICE Collaboration, Physics Performance Report Vol. II, CERN/LHCC 2005-030.

3. J. Dunlop, these proceedings.

4. M. Mangano, P. Nason and G. Ridolfi, Nucl. Phys. B 373, 295 (1992).

5. Yu.L. Dokshitzer and D.E. Kharzeev, Phys. Lett. B 519, 199 (2001).

6. N. Armesto, C.A. Salgado and U.A. Wiedemann, Phys. Rev. D 69, 114003 (2004).

7. M. Djordjevic and M. Gyulassy, Phys. Lett. B 560, 37 (2003); Nucl. Phys. A 733, 265 (2004).

8. B. W. Zhang, E. Wang and X. N. Wang, Phys. Rev. Lett. 93, 072301 (2004).

9. R. Baier, Y. L. Dokshitzer, A. H. Mueller, S. Peigne and D. Schiff, Nucl. Phys. B 484, 265 (1997).

10. C. A. Salgado and U. A. Wiedemann, Phys. Rev. D68, 014008 (2003).

11. N. Armesto, A. Dainese, C.A. Salgado and U.A. Wiedemann, Phys. Rev. D 71, 054027 (2005).

12. A. Dainese, C. Loizides and G. Paić, Eur. Phys. J. C 38, $461(2005)$.

13. C. Loizides, these proceedings.

14. A. Dainese, N. Armesto, M. Cacciari, C. A. Salgado and U. A. Wiedemann, arXiv:hep-ph/0601107

15. N. Armesto, M. Cacciari, A. Dainese, C. A. Salgado and U. A. Wiedemann, Phys. Lett. B 637, 362 (2006).

16. M. Cacciari, P. Nason and R. Vogt, Phys. Rev. Lett. 95, 122001 (2005).

17. S. S. Adler et al., PHENIX Coll., Phys. Rev. Lett. 96, 032001 (2006).

18. S. S. Adler et al., PHENIX Coll., Phys. Rev. Lett. 96, 032301 (2006)

19. J. Bielcik et al., STAR Coll., arXiv:nucl-ex/0511005

20. S. Wicks, W. Horowitz, M. Djordjevic and M. Gyulassy, arXiv:nucl-th/0512076

21. X. N. Wang, arXiv:nucl-th/0604040

22. R. Guernane et al., ALICE Internal Note, ALICE-INT2005-018 (2005).

23. Z. Conesa del Valle, these proceedings. 
24. N. Carrer, A. Dainese and R. Turrisi, J. Phys. G 29, 575 (2003).

25. A. Dainese, Ph.D. Thesis (2003), arXiv:nucl-ex/0311004

26. K.J. Eskola, V.J. Kolhinen, C.A. Salgado, Eur. Phys. J. C 9, 61 (1999). 
